\title{
Unaltered Hepatic Oxidative Phosphorylation and Mitochondrial Permeability Transition in Wistar Rats Treated With Nimesulide: Relevance for Nimesulide Toxicity Characterization
}

\author{
António J. Moreno, ${ }^{1}$ Paulo J. Oliveira, ${ }^{2}$ Carlos D. Nova,${ }^{3}$ Ana R. Álvaro, ${ }^{2}$ \\ Renata A. Moreira, ${ }^{3}$ Sandra M. D. Santos, ${ }^{4}$ and Tice Macedo ${ }^{4}$ \\ ${ }^{1}$ Institute of Marine Research, Department of Zoology, University of Coimbra, Coimbra, Portugal \\ ${ }^{2}$ Center for Neurosciences and Cellular Biology, Department of Zoology, University of Coimbra, Coimbra, Portugal; E-mail: pauloliv@ci.uc.pt \\ ${ }^{3}$ Department of Zoology, University of Coimbra, Coimbra, Portugal \\ ${ }^{4}$ Institute of Pharmacology and Experimental Therapeutics, School of Medicine, University of Coimbra, Coimbra, Portugal
}

Received 8 November 2006; revised 12 February 2007; accepted 14 February 2007

\begin{abstract}
Nonsteroidal anti-inflammatory drugs have been associated with hepatotoxicity in susceptible patients. One such example is nimesulide, a preferential cyclooxygenase 2-inhibitor, widely used for the treatment of inflammation and pain. It was suggested that nimesulide could exert its hepatotoxicity by altering hepatic mitochondrial function, which was demonstrated in vitro. The objective of this study was to verify whether liver mitochondria isolated from rats treated with doses of nimesulide well above therapeutic levels possessed decreased calcium tolerance and oxidative phosphorylation, which indicates in vivo nimesulide mitochondrial toxicity. Male and female rats received nimesulide or its vehicle twice daily, for 5 days, and were killed on the seventh day for the isolation of liver mitochondria. Mitochondrial respiration, transmembrane electric potential, and calcium tolerance were characterized in all experimental groups. Nimesulide had no effect on liver mitochondrial function. Indexes of mitochondrial integrity, calcium loading capacity, and oxidative phosphorylation efficiency were unchanged between liver mitochondria from treated and control animals. In the animals tested, no evidence of degraded mitochondrial function due to nimesulide administration could be found. The results corroborate the notion that despite recognized in vitro mitochondrial toxicity, nimesulide does not cause detectable mitochondrial dysfunction in Wistar rats, even
\end{abstract}

Correspondence to: Paulo J. Oliveira.

Contract Grant Sponsor: Helsinn Healthcare SA, Switzerland.

Contract Grant Sponsor: Portuguese Foundation for Science and Technology.

Contract Grant Number: SFRH/BPD/8359/2002.

(C) 2007 Wiley Periodicals, Inc.

(2)WILEY

InterScience when administered in much higher concentrations than those known to have anti-inflammatory effects. (C) 2007 Wiley Periodicals, Inc. J Biochem Mol Toxicol 21:53-61, 2007; Published online in Wiley InterScience (www.interscience.wiley.com). DOI 10:1002/jbt.20159

KEYWORDS: Nimesulide; Liver Mitochondria; Oxidative Phosphorylation; Mitochondrial Permeability Transition; Wistar Rat

\section{INTRODUCTION}

Nimesulide (N-[4-nitro-2-phenoxyphenyl]-methanesulfonamide) is a widely used nonsteroidal antiinflammatory drug (NSAID) that, similarly to other NSAIDs, has been associated with rare, but sometimes severe, hepatic adverse reactions. The relevant frequency of adverse reactions is comparable with that of other NSAIDs, which are known to be associated with hepatotoxicity $[1,2]$. The hepatic events reported during treatment with nimesulide include asymptomatic and reversible elevation of liver enzymes [1,3-5], acute hepatitis associated with hepatocellular necrosis [6], cholestasis [7], and some isolated cases of fatal acute hepatic failure $[8,9]$.

So far, the molecular mechanisms underlying the hepatotoxicity of nimesulide have not been fully elucidated. Nimesulide is extensively metabolized in the liver, predominantly to the metabolite 4hydroxy-nimesulide [10]. Nimesulide-induced hepatocellular damage usually presents with necrosis in the 
centrilobular region, where cytochrome $\mathrm{P}_{450}$ activity is highest [11]. Genetic predisposition could favor the production of uncommon metabolites that can either exert a toxic effect directly or induce an immunological reaction by forming adducts with the cytochrome $\mathrm{P}_{450}$ complex, which are subsequently internalized by B lymphocytes [12]. As opposed to what has been described, studies on the viability and growth of the human hepatoma cell line HepG2 showed that nimesulide or its metabolites exhibited no appreciable cytotoxic activity [13].

Mitochondria are a potential target of drug toxicity. In fact, it is well known that a large number of natural, commercial, pharmaceutical, and environmental chemicals manifest their toxicity by interfering with mitochondrial bioenergetics $[14,15]$. Several in vitro studies suggest that one of the reasons for the hepatic toxicity of nimesulide may be the impairment of liver mitochondrial function. Previous in vitro studies carried out on isolated mitochondria and isolated hepatocytes have shown various mitochondrial effects. For example, nimesulide uncouples mitochondria through a protonophoretic mechanism and decreases the phosphorylation index (ADP/O ratio) and the respiratory control ratio (RCR) [16-20].

Probably one of the most relevant aspects of nimesulide-induced hepatotoxicity is the ability of the drug to induce mitochondrial permeability transition (MPT). In fact, Mingatto et al. [18] demonstrated that nimesulide acts as a potent protonophoretic uncoupler and $\mathrm{NAD}(\mathrm{P}) \mathrm{H}$ oxidant on isolated rat liver mitochondria, inducing calcium efflux through MPT induction. MPT is a phenomenon related to the formation and opening of protein pores (the MPT pores) in the inner mitochondrial membrane [21]. MPT can be caused by excessive mitochondrial calcium accumulation and oxidative stress generation [22], leading ultimately to cell death [23], which could be an important contribution for the pathophysiology of nimesulide-induced hepatotoxicity. A temporal relation between mitochondrial depolarization, generation of superoxide radical, and induction of the MPT by nimesulide was established by Tay et al. [24]. According to these authors, mitochondrial uncoupling induced by nimesulide is the trigger for induction of the MPT, which further leads to the generation of oxidative stress.

Despite extensive knowledge of the effects of nimesulide on isolated liver mitochondria, data are lacking regarding hepatic mitochondrial function of nimesulide-treated rats, namely and most importantly, the susceptibility of liver mitochondria from treated animals to undergo MPT. The objective of the present study was to verify if nimesulide administration to Wistar rats could induce early mitochondrial alterations comparable to those observed in in vitro studies.
In agreement with previous in vitro work, the initial hypothesis was that liver mitochondria isolated from nimesulide-treated rats showed decreased tolerance to calcium (enhanced MPT) and altered respiratory parameters.

\section{MATERIALS AND METHODS}

\section{Chemicals}

Nimesulide was provided by Helsinn Healthcare SA (and dissolved in absolute DMSO). Bovine serum albumin (BSA), rotenone, $\mathrm{N}-(2-$ hydroxyethyl)piperazine- $N^{\prime}$-(2-ethanesulfonic acid) (HEPES), cyclosporin $\mathrm{A}$, and ethylene glycol-bis $(\beta-$ aminoethyl ether)- $N, N, N^{\prime}, N^{\prime}$-tetraacetic acid (EGTA) were purchased from Sigma Chemical Co. (St. Louis, $\mathrm{MO})$. Tetraphenylphosphonium $\left(\mathrm{TPP}^{+}\right)$and sucrose were from Merck (Darmstadt, Germany). All other reagents and chemicals were commercial products of the highest purity grade available. Solutions were prepared in deionized ultra-pure water.

\section{Animals}

Wistar rats (250-300 g) were used for the experiments and kept under controlled light $(12 \mathrm{~h} / 12 \mathrm{~h}$ day/night cycle), temperature $\left(22-24^{\circ} \mathrm{C}\right)$, and humidity $(50 \%-60 \%)$ conditions, and with free access to water (pH 5.5) and food. The experiments reported here were carried out in accordance with the National Requirements for Vertebrate Animal Research and in accordance with the European Convention for the Protection of Animals Used for Experimental and Other Scientific Purposes. Twelve animals were randomly divided into four groups (each $n=3$ ) of six male and six female Wistar rats as follows: (i) control male animals $(n=3)$ receiving DMSO (vehicle) twice daily (injected i.p.) for 5 days and killed on the seventh day for isolating liver mitochondria; (ii) treated male rats $(n=3)$ receiving i.p. injections of nimesulide $50 \mathrm{mg} / \mathrm{kg}$ twice daily for 5 days and killed on the seventh day for isolating liver mitochondria; (iii) control female animals $(n=3)$ receiving DMSO (vehicle) twice daily (injected i.p.) for 5 days and killed on the seventh day for isolating liver mitochondria; (iv) treated female rats $(n=3)$ receiving i.p. injections (total volume of administration: $0.1 \mathrm{~mL}$ ) of nimesulide $50 \mathrm{mg} / \mathrm{kg}$ twice daily for 5 days and killed on the seventh day for isolating liver mitochondria. In the same day of experiments, one treated and one control animal of the same gender were used. The nimesulide dosage used in the present study is much higher than those known to have anti-inflammatory action in man as the objective was to verify potential toxic 
effects on mitochondrial function. The usual human therapeutic regimen is $100 \mathrm{mg}$ nimesulide taken twice daily for 5 days [25]. In the present study, we used the same length of time and a dosage that is about 30 times higher than the maximum recommended dose administered to a $70-\mathrm{kg}$ human.

\section{Isolation of Rat Liver Mitochondria}

Rat liver mitochondria were isolated by conventional differential centrifugation [26] from the livers of overnight-fasted rats, with slight modifications. Rats were killed by decapitation, and their livers were harvested and rinsed with an ice-cold buffer containing $250 \mathrm{mM}$ sucrose, $10 \mathrm{mM}$ HEPES, $1 \mathrm{mM}$ EGTA, and $0.1 \%$ defatted BSA. The $\mathrm{pH}$ was adjusted to 7.4 with $\mathrm{KOH}$. Livers were trimmed and homogenized for $1 \mathrm{~min}$ with a motor-driven Teflon Potter homogenizer in the presence of ice-cold buffer $(7 \mathrm{~g} / 50 \mathrm{~mL})$. Liver homogenate was centrifuged at $800 \times g$ for 10 min (Sorvall RC-5C, Plus, SS 34 rotor, $4^{\circ} \mathrm{C}$ ) and its supernatant at $10,000 \times g$ for $10 \mathrm{~min}$. The mitochondrial pellet was resuspended using a paintbrush and centrifuged twice at 10,000 $\times g$ for $10 \mathrm{~min}$ to obtain a final mitochondrial suspension. EGTA and defatted BSA were omitted from the final washing medium, which was adjusted to $\mathrm{pH}$ 7.2. Mitochondrial protein was determined by the biuret method using BSA as a standard. The mitochondrial suspension $(\approx 30-40 \mathrm{mg}$ protein $/ \mathrm{mL}$ ) was kept on ice during experiments, which were carried out after a 20-min recovery and within $5 \mathrm{~h}$.

\section{Mitochondrial Oxygen Consumption Measurements}

Oxygen consumption was measured using a Clarktype oxygen electrode (Yellow Springs Instruments, $\mathrm{OH}$ ) connected to a recorder (Kipp \& Zonen, BD 112) in a thermostated water-jacketed sealed glass chamber with constant magnetic stirring, at $25^{\circ} \mathrm{C}$. Reactions were conducted in $1 \mathrm{~mL}$ of medium containing $125 \mathrm{mM}$ sucrose, $65 \mathrm{mM} \mathrm{KCl}, 2.5 \mathrm{mM} \mathrm{MgCl} 2,5 \mathrm{mM}$ $\mathrm{KH}_{2} \mathrm{PO}_{4}, 5 \mathrm{mM}$ HEPES (pH 7.2), supplemented with $3 \mu \mathrm{M}$ rotenone. The reactions were initiated by adding potassium succinate to a final concentration of $5 \mathrm{mM}$. State 2 respiration was monitored for at least $2 \mathrm{~min}$ prior to adding ADP $(0.1 \mathrm{mM})$ to initiate state 3 respiration. State 4 respiration followed exhaustion of ADP. $\mathrm{RCRs}$ and $\mathrm{ADP} / \mathrm{O}$ ratios were determined according to Estabrook [27]. The scale of oxygen uptake was calibrated according to the oxygen consumed by submitochondrial particles after addition of titrated solutions of $\mathrm{NADH}$.

\section{Measurements of Mitochondrial Transmembrane Electrical Potential}

The mitochondrial membrane potential $(\Delta \Psi)$ was monitored indirectly based on the activity of the lipophilic cation tetraphenylphosphonium $\left(\mathrm{TPP}^{+}\right)$using a $\mathrm{TPP}^{+}$-selective electrode prepared in our laboratory, in combination with a $\mathrm{Ag} / \mathrm{AgCl}$-saturated reference electrode (Tacussel, Model MI 402) [28]. Both the $\mathrm{TPP}^{+}$electrode and the reference electrode were inserted into an open vessel with magnetic stirring and connected to a $\mathrm{pH}$ meter (Jenway, Model 3305). The signals were fed to a potentiometric recorder (Kipp \& Zonen, Model BD 121). Mitochondria were incubated in the reaction medium consisting of $125 \mathrm{mM}$ sucrose, $65 \mathrm{mM} \mathrm{KCl}, 2.5 \mathrm{mM} \mathrm{MgCl} 2,5 \mathrm{mM} \mathrm{KH} \mathrm{PO}_{4}, 5 \mathrm{mM}$ HEPES (pH 7.2), supplemented with $3 \mu \mathrm{M}$ rotenone, containing $3 \mu \mathrm{M} \mathrm{TPP}$. The reaction was stirred continuously and the temperature maintained at $25^{\circ} \mathrm{C}$. The experiments were started by adding $5 \mathrm{mM}$ succinate to mitochondria in suspension at $1 \mathrm{mg}$ protein $/ \mathrm{mL}$. ADP or $\mathrm{Ca}^{2+}$ was added and $\Delta \Psi$ fluctuations recorded after a steady-state distribution of $\mathrm{TPP}^{+}$had been reached (around 2 min of recording). The experiments with $\mathrm{Ca}^{2+}$ were carried out in a medium consisting of $0.2 \mathrm{M}$ sucrose, $10 \mathrm{mM}$ Tris-MOPS (pH 7.4), $1 \mathrm{mM} \mathrm{KH}_{2} \mathrm{PO}_{4}$, and $10 \mu \mathrm{M}$ EGTA, supplemented with $2 \mu \mathrm{M}$ rotenone.

The $\Delta \Psi$ was estimated as indicated by Kamo et al. [27] from the following equation (at $25^{\circ} \mathrm{C}$ ): $\Delta \Psi=59 \times$ $\log (v / V)-59 \times \log (10 \Delta E / 59-1)$, where $v, V$, and $\Delta E$ stand for mitochondrial volume, volume of the incubation medium, and deflection of the electrode potential from the baseline, respectively. A matrix volume of $1.1 \mu \mathrm{L}$ per mg protein was assumed. No correction was made for the "passive" binding of $\mathrm{TPP}^{+}$to the mitochondrial membranes, since the purpose of the experiments was to show relative changes in potentials rather than absolute values. As a consequence, a slight overestimation of the $\Delta \Psi$ values is anticipated.

\section{Measurement of Mitochondrial Swelling}

Changes in the mitochondrial osmotic volume were followed by monitoring the decrease in absorbance (light scattering) at $540 \mathrm{~nm}$ with a Jasco V-560 spectrophotometer as previously described [26]. The reactions were performed with $1 \mathrm{mg}$ mitochondrial protein in $2 \mathrm{~mL}$ of a medium consisting of $0.2 \mathrm{M}$ sucrose, $10 \mathrm{mM}$ Tris-MOPS (pH 7.4), 1 mM KH${ }_{2} \mathrm{PO}_{4}$, and $10 \mu \mathrm{M}$ EGTA, supplemented with $2 \mu \mathrm{M}$ rotenone. The reaction was stirred continuously and the temperature was maintained at $25^{\circ} \mathrm{C}$. Mitochondria were energized with $5 \mathrm{mM}$ succinate for $2 \mathrm{~min}$ before adding $60 \mathrm{nmol}$ $\mathrm{CaCl}_{2}$. Cyclosporin $\mathrm{A}(1 \mu \mathrm{M}$, in ethanol) was added just before adding succinate. 


\section{Mitochondrial $\mathrm{Ca}^{2+}$ Fluxes}

Mitochondrial $\mathrm{Ca}^{2+}$ fluxes were measured by monitoring the changes in $\mathrm{Ca}^{2+}$ concentration in the reaction medium using a $\mathrm{Ca}^{2+}$-selective electrode according to previously described procedures [29]. The reactions were conducted in an open vessel with magnetic stirring in $1 \mathrm{~mL}$ of the reaction medium composed of $0.2 \mathrm{M}$ sucrose, $10 \mathrm{mM}$ Tris-MOPS ( $\mathrm{pH}$ 7.4), $1 \mathrm{mM}$ $\mathrm{KH}_{2} \mathrm{PO}_{4}$, and $10 \mu \mathrm{M}$ EGTA, supplemented with $2 \mu \mathrm{M}$ rotenone. Mitochondria (1 mg) were energized with $5 \mathrm{mM}$ succinate after $2 \mathrm{~min}$ of $\mathrm{Ca}^{2+}$ addition $(60 \mathrm{nmol})$.

\section{Statistical Analysis}

The results are presented as mean \pm SEM for the number of experiments indicated in the legends to the figures. Multiple comparisons were performed using one-way ANOVAs.

\section{RESULTS}

The respiratory control ratio (RCR) is a measure of the dependence of the respiratory rate on oxidative phosphorylation. A decrease of the RCR ratio generally indicates that the structural integrity of mitochondria has been affected. On the other hand, the ADP/O ratio is a measure of the efficiency of mitochondrial oxidative phosphorylation. RCR and ADP/O ratios of liver mitochondria isolated from either male or female nimesulide-treated rats are not different relative to the controls (Table 1), which indicates that nimesulide treatment neither affects the mitochondrial respiration nor does it impair the efficiency of ATP synthesis (as indicated by the ADP/O ratio) of isolated liver mitochondria. The values of the RCR are around 6, denoting a good mitochondrial preparation, which is further confirmed by the proximity to 2 of the ADP/O values.

To obtain further insight into the effects of nimesulide on the capacity of mitochondria to use energy arising from oxidative metabolism, $\Delta \Psi$ fluctuations

TABLE 1. Effect of Nimesulide on RCR and ADP/O Ratios of Rat Liver Mitochondria Isolated From Control and Nimesulide-Treated Rats ${ }^{a}$

\begin{tabular}{lcc}
\hline & $R C R$ & $A D P / O$ \\
\hline Control (male) & $6.00 \pm 0.04$ & $1.91 \pm 0.02$ \\
Nimesulide (male) & $5.99 \pm 0.04$ & $1.89 \pm 0.01$ \\
Control (female) & $5.96 \pm 0.04$ & $1.90 \pm 0.02$ \\
Nimesulide (female) & $5.97 \pm 0.04$ & $1.89 \pm 0.01$ \\
\hline
\end{tabular}

${ }^{a}$ Data are means \pm SEM obtained from three different mitochondrial preparations for each experimental group. For each mitochondrial preparation, assays were performed in triplicate.
TABLE 2. Effect of Nimesulide Treatment on Mitochondrial Transmembrane Potential $(\Delta \Psi)$ Fluctuations $^{a}$

\begin{tabular}{lcccc}
\hline & \multicolumn{3}{c}{$\Delta \Psi(-m V)$} & \\
\cline { 2 - 3 } & $\begin{array}{c}\text { Succinate } \\
\text { Energization }\end{array}$ & $\begin{array}{c}\text { ADP } \\
\text { Depolarization }\end{array}$ & Repolarization & Lag Phase (s) \\
\hline $\begin{array}{l}\text { Control } \\
\text { (male) }\end{array}$ & $229 \pm 0.6$ & $31.0 \pm 0.83$ & $30.8 \pm 0.8$ & $61 \pm 2.2$ \\
$\begin{array}{l}\text { Nimesulide } \\
\text { (male) }\end{array}$ & $227 \pm 1.0$ & $29.6 \pm 0.8$ & $29.5 \pm 0.9$ & $62.5 \pm 2.1$ \\
$\begin{array}{l}\text { Control } \\
\text { (female) }\end{array}$ & $228 \pm 0.6$ & $28.8 \pm 0.5$ & $27.3 \pm 0.4$ & $57.8 \pm 1.3$ \\
$\begin{array}{l}\text { Nimesulide } \\
\text { (female) }\end{array}$ & $228 \pm 0.5$ & $29.8 \pm 0.8$ & $28.3 \pm 0.6$ & $58.6 \pm 1.2$ \\
\hline
\end{tabular}

${ }^{a}$ Table shows the average response of membrane potential developed with succinate, the decrease in membrane potential after ADP addition, the lag phase that precedes repolarization, and the repolarization value after ADP phosphorylation. There are no statistically significant differences when compared to controls. Data are mean \pm SEM obtained from three different mitochondrial preparations (with assays in quadruplicate for each preparation).

associated with mitochondrial respiration and the phosphorylation cycle induced by ADP were also investigated. Mitochondria isolated from control and nimesulide-treated animals (male or female) show no difference relatively to $\Delta \Psi$ fluctuations (Table 2). Upon energization with succinate, all groups of mitochondria (isolated from control and treated animals) developed the same $\Delta \Psi$ (around $-230 \mathrm{mV}$ ). The depolarization that follows the addition of ADP, the repolarization rate, and the recovery of $\Delta \Psi$ after the phosphorylation cycle are also not affected after nimesulide treatment. Finally, the lag phase that precedes the repolarization of the mitochondrial membrane is not affected by the treatment with nimesulide. As the lag phase represents the time required for the phosphorylation of added ADP, nimesulide does not affect the time required for the phosphorylation of ADP (which is in accordance with the unchanged ADP/O values). After the phosphorylation cycle, the nucleotide contents (ATP, ADP, and AMP) were also analyzed by HPLC. The energetic charge of mitochondria isolated from control rats and nimesulide-treated rats are similar (data not shown). This result confirms that nimesulide does not alter the phosphorylation efficiency of mitochondria, as described previously.

The nitroreductive metabolism of nimesulide involves redox cycling. The resulting production of reactive oxygen species (namely superoxide anion molecules) can potentially lead to intracellular oxidative stress and result in mitochondrial dysfunction through the opening of the MTP pore. Following this idea, the possible in vivo effects of nimesulide on the opening of the mitochondrial permeability transition pore was also investigated. Figure 1 represents a typical recording from swelling experiments carried out in mitochondria from male Wistar rats. The 


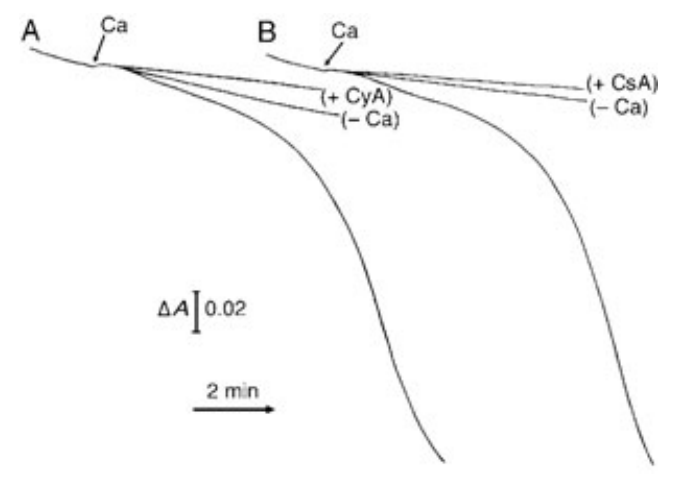

FIGURE 1. Effect of nimesulide treatment on calcium-dependent induction of the MPT. Rat liver mitochondria (1 mg) were incubated at $25^{\circ} \mathrm{C}$ in $2 \mathrm{~mL}$ of sucrose $200 \mathrm{mM}$, Tris-MOPS $10 \mathrm{mM}$ (pH 7.2), $\mathrm{KH}_{2} \mathrm{PO}_{4}$ $1 \mathrm{mM}$, and EGTA $10 \mu \mathrm{M}$, supplemented with $2 \mu \mathrm{M}$ rotenone. Mitochondria were energized with $5 \mathrm{mM}$ succinate before adding $\mathrm{CaCl}_{2}$. Light scattering was monitored continuously at $540 \mathrm{~nm}$, which served as the basis for assessing changes in mitochondrial volume. Shown here is the result of experiments involving male rats. Results obtained with female rats do not differ (data not shown). The traces are typical of three different preparations, with assays done in duplicate. (A) Mitochondria isolated from vehicle (DMSO)-treated rats (control) and (B) mitochondria isolated from nimesulide-treated rats. CsA: Cyclosporin A.

MTP pore was induced by the addition of calcium $(60 \mathrm{nmol})$ to succinate-energized mitochondria. As seen in Figure 1B, mitochondria isolated from nimesulidetreated rats show a susceptibility to the opening of the MPT pore comparable to those isolated from control rats (Figure 1A). Results obtained with female rats were identical (data not shown). Cyclosporin A, the specific inhibitor of the MPT pore [30], completely inhibited swelling in all experimental groups (Figure 1 and data not shown).

$\Delta \Psi$ collapse and calcium efflux associated with MPT pore opening were also studied in both control and nimesulide-treated rats. The results presented in Figure 2 illustrate the fluctuations of mitochondrial $\Delta \Psi$ associated with the addition of two successive pulses of $30 \mathrm{nmol}$ of calcium to succinate-energized mitochondria from male rats. Adding $30 \mathrm{nmol}$ of calcium to energized mitochondria (of control and treated animals) caused a transient depolarization of the membrane potential (Figures 2A and 2B). The membrane returned to near the original potential within less than $1 \mathrm{~min}$. However, after a second pulse of $30 \mathrm{nmol}$ calcium, the mitochondria were unable to sustain membrane potential and underwent depolarization. The $\Delta \Psi$ collapse is completely prevented by cyclosporin A (in both control and treated rats; Figure 2C), which indicates that the depolarization is related to the induction of the MPT pore. Results obtained with female rats are very similar (data not shown). Again, no differences were found among experimental groups.

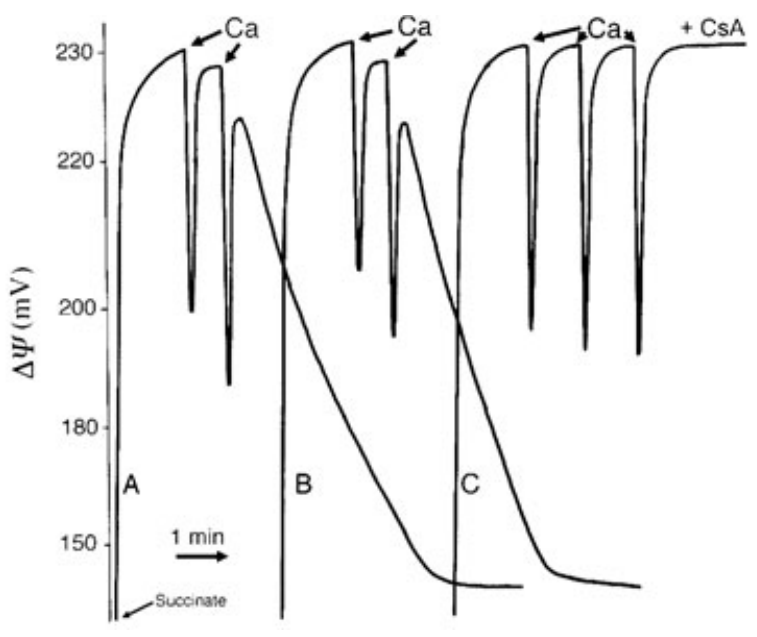

FIGURE 2. Effect of nimesulide treatment on mitochondrial $\Delta \Psi$ associated with $\mathrm{Ca}^{2+}$-induced depolarization of succinate-energized mitochondria. Freshly isolated rat liver mitochondria $(1 \mathrm{mg})$ were incubated at $25^{\circ} \mathrm{C}$ in $1 \mathrm{~mL}$ of sucrose $200 \mathrm{mM}$, Tris-MOPS $10 \mathrm{mM}$ ( $\mathrm{pH}$ 7.2), $\mathrm{KH}_{2} \mathrm{PO}_{4} 1 \mathrm{mM}$, EGTA $10 \mu \mathrm{M}$ supplemented with $2 \mu \mathrm{M}$ rotenone. $\Delta \Psi$ was recorded continuously using a $\mathrm{TPP}^{+}$-selective electrode linked to a suitable recorder. Energization was initiated by adding $5 \mathrm{mM}$ succinate followed by two pulses of $30 \mathrm{nmol} \mathrm{CaCl}$. The second pulse of calcium induced the MPT (A and B). Note that cyclosporin A (CsA) prevented the depolarization induced by $\mathrm{Ca}^{2+}$ in both experimental groups (C [recordings from both groups were superimposed]). Shown here is the result of experiments involving male rats. Results obtained with female rats do not differ (data not shown). The traces are typical of three different preparations, with assays done in duplicate. (A) Mitochondria isolated from vehicle (DMSO)-treated rats (control), (B) mitochondria isolated from nimesulide-treated rats, and (C) mitochondria isolated from male nontreated animals or treated animals in the presence of $1 \mu \mathrm{M}$ of cyclosporin A.

The lack of effect of nimesulide in the induction of mitochondrial permeability transition is further evidenced by following calcium fluxes through the mitochondrial membrane. Energized mitochondria accumulate calcium from the medium for a certain period of time. The rapid uptake of calcium following energization with succinate was followed by a dynamic steady state corresponding to the equilibrium between the influx and the efflux of calcium. The results reported in Figure 3 show that mitochondria isolated from male nimesulide-treated rats (Figure 3B) and male control animals (Figure 3A) accumulated approximately the same amount of calcium, and released the preaccumulated calcium due to the induction of the MPT after the same lag time. Cyclosporin A was able to prevent calcium release to the external buffer in both experimental groups (Figure 3C). As with previous data, results obtained with female animals were duplications of the results obtained with male animals (data not shown).

Finally, we also followed variations of mitochondrial oxygen consumption upon calcium additions. 


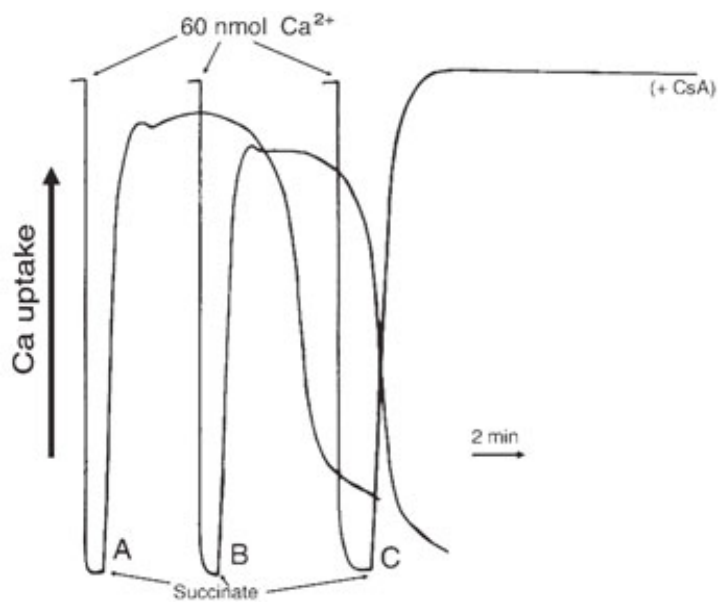

FIGURE 3. Effect of nimesulide treatment on mitochondrial $\mathrm{Ca}^{2+} \mathrm{re}^{-}$ lease associated with permeability transition pore opening. Freshly isolated rat liver mitochondria $(1 \mathrm{mg})$ were incubated at $25^{\circ} \mathrm{C}$ in $1 \mathrm{~mL}$ of sucrose $200 \mathrm{mM}$, Tris-MOPS $10 \mathrm{mM}$ (pH 7.2), $\mathrm{KH}_{2} \mathrm{PO}_{4}$ $1 \mathrm{mM}$, and EGTA $10 \mu \mathrm{M}$ supplemented with $2 \mu \mathrm{M}$ rotenone. Energization was initiated by adding $5 \mathrm{mM}$ succinate after the addition of $60 \mathrm{nmol}$ of $\mathrm{CaCl}_{2}$. The uptake of $\mathrm{Ca}^{2+}$ and release of sequestered $\mathrm{Ca}^{2+}$ by mitochondria were continuously recorded using a $\mathrm{Ca}^{2+}$ selective electrode linked to a suitable recorder. Note that cyclosporin A prevented the release of $\mathrm{Ca}^{2+}(\mathrm{C})$, which confirms the involvement of the mitochondrial permeability transition in this phenomenon. (A) Mitochondria isolated from vehicle (DMSO)-treated rats (control), (B) mitochondria isolated from nimesulide-treated rats, and (C) mitochondria isolated from male nontreated or treated animals in the presence of $1 \mu \mathrm{M}$ of cyclosporin A (CsA [recordings from both groups were superimposed]). Shown here is the result of experiments involving male rats. Results obtained with female rats were similar (data not shown). The traces are typical of three different preparations, with assays done in duplicate.

There was no difference between experimental groups regarding calcium-induced mitochondrial stimulation of respiration (data not shown). Cyclosporin A again prevented uncoupling of respiration due to calciuminduced MPT (data not shown).

\section{DISCUSSION}

Nimesulide is a well-tolerated nonsteroidal antiinflammatory drug (NSAID) with a wide range of clinical uses. However, its use has raised some concern regarding its potential hepatotoxicity in susceptible patients [31]. The exact biochemical mechanisms of nimesulide toxicity have not been fully elucidated, yet some studies have suggested an important role for nimesulide-induced mitochondrial dysfunction in the pathogenesis of liver injury. In fact, in vitro studies have demonstrated that nimesulide acts as a potent protonophoretic uncoupler and $\mathrm{NAD}(\mathrm{P}) \mathrm{H}$ oxidant in isolated rat liver mitochondria [18], and in isolated rat hepatocytes [20]. These authors also demon- strated that nimesulide promotes mitochondrial $\mathrm{Ca}^{2+}$ efflux through the mitochondrial permeability transition (MPT) pore within a concentration range claimed by the authors to be potentially reached in vivo (approximately $5 \mu \mathrm{M}$ ). The peak plasma concentrations of nimesulide in humans are indeed in the range $10-20 \mu \mathrm{M}$ [24], but considering that the drug is $99 \%$ bound to plasma protein, the unbound, bioavailable fraction is significantly lower. Nevertheless, these observations raised the possibility that nimesulide, through its capability to interfere with mitochondrial bioenergetics, has the ability to interfere with the energy production and $\mathrm{Ca}^{2+}$ homeostasis in the liver cell, which in turn may constitute a relevant mechanism for the reported nimesulide-induced liver toxicity. Later, Tay et al. [24] described that mitochondrial uncoupling induced by nimesulide is actually the trigger for induction of the MPT, which further leads to the generation of oxidative stress on isolated liver mitochondria.

The rat is generally regarded as a suitable model for NSAIDs damage in humans $[32,33]$ In this study, we investigated whether mitochondria isolated from the livers of rats treated with supratherapeutic doses of nimesulide (50 mg/kg, twice daily, for 5 days) showed early signs of dysfunction, including a decreased calciumloading capacity, which has been shown to be a reliable marker for drug-induced mitochondrial toxicity [34]. In addition, we tried to demonstrate whether previous conclusions taken from in vitro studies of mitochondria can be extrapolated to in vivo situations.

Pharmacokinetic studies with $100 \mathrm{mg}$ nimesulide administrated twice daily for 5 days in humans revealed that the drug is rapidly distributed, extensively bound to albumin, and eliminated with a terminal half-life of about $4 \mathrm{~h}$ [25]. Nimesulide is oxidatively metabolized via liver cytochrome $\mathrm{P}_{450}$ mainly to the 4-hydroxy-metabolite (4-OH-NME), which has similar pharmacological properties to the parent drug, although with a lower potency. The period that elapses before the appearance of the metabolite in the circulation is short (approximately $1 \mathrm{~h}$ ) and half-life $\left(t_{1 / 2}\right)$ is between 3.2 and $8 \mathrm{~h}$. The plasma elimination is progressive, with a $t_{1 / 2}$ in plasma of $2-5 \mathrm{~h}$ for the parent drug and $3-9 \mathrm{~h}$ for $4-\mathrm{OH}-\mathrm{NME}$, thus allowing for convenient 100-mg twice-daily dosage without any evidence of accumulation [35]. The elimination of nimesulide is not influenced by age or gender, but its use is contraindicated in patients with hepatic impairment [36]. The usual therapeutic regimen is $100 \mathrm{mg}$ twice daily, with discontinuation of the administration after 5 days. The pharmacokinetic profiles of nimesulide in humans and rats appear to be similar $[25,37,38]$.

Data from the present investigation demonstrate that the RCR and the phosphorylation index (ADP/O) of liver mitochondria isolated from nimesulide-treated 
rats were not affected, which means that the structural integrity and the phosphorylation capability of mitochondria were maintained (Table 1). These results indicate that mitochondrial bioenergetic function was not impaired by nimesulide.

To further confirm that nimesulide treatment did not impair hepatic mitochondria, the transmembrane electric potential (Table 2) associated with mitochondrial respiration and the phosphorylation cycle induced by ADP was also measured. The study of $\Delta \Psi$ is essential for an integrated appraisal of the mitochondrial function, since it reflects basic useful energetic relationships. Nimesulide treatment affected neither the energization by succinate nor the $\Delta \Psi$ fluctuations linked to the phosphorylation cycle, confirming that the drug treatment did not interfere with the respiratory chain or with the phosphorylation system of mitochondria, irrespective of whether they had been isolated from male or female animals.

Mitochondrial dysfunction can also be triggered by stress-induced opening of the MPT pore. Induction of the MPT is implicated in the mechanisms of cell injury caused by a number of xenobiotics, many of which behave as oxidants $[39,40]$. In fact, the opening of the MPT pore upon increased oxidative stress is well characterized. It is now well recognized that the MPT is closely involved in cell death either by necrosis or by apoptosis $[22,23,39,41]$. In this regard, there is evidence that nimesulide can induce intracellular oxidative stress in vitro; its capability for redox cycling can produce superoxide anion and pose an oxidative stress [24,31,42]. Tay et al. [24] demonstrated that the oxidative stress of isolated liver mitochondria exposed to nimesulide was a consequence of MPT pore opening.

In vivo effects of nimesulide on mitochondrial calcium handling have not been analyzed so far. Bearing this in mind, we investigated the effect of nimesulide treatment upon calcium-dependent mitochondrial permeability transition. A hallmark of the MPT is the highamplitude swelling induced by calcium in the presence of phosphate. In our assays, the addition of calcium to energized mitochondria caused a large-amplitude decrease in light scattering, typical of the mitochondrial swelling induced by the opening of the MPT pore in liver mitochondria. The inhibition by Cyclosporin A is evidence for the induction of the MPT, which is attributed to the $\mathrm{Ca} / \mathrm{Pi}$-induced stimulation of hydrogen peroxide generation [43]. The fact that the curve profile for mitochondria isolated from control and treated animals (of both genders) is the same (Figure 1) indicates that the susceptibility for the induction of MPT is unaltered.

Confirming the previous results, we also verified that mitochondria isolated from either nimesulidetreated (of both sexes) or nontreated animals show the same profile of $\Delta \Psi$ fluctuations, indicating again that the susceptibility for the calcium-induced MPT was not altered (Figure 2). The final evidence arises from determination of extramitochondrial calcium movements using a $\mathrm{Ca}^{2+}$-selective electrode. Again, results confirm the previous data (Figure 3). Indeed, liver mitochondria isolated from nimesulide-treated and control animals were able to accumulate the same amount of calcium and to retain the calcium for the same length of time before its release to the reaction medium. The result indicates that nimesulide did not confer any particular susceptibility to the induction of MPT. Cyclosporin A was able to inhibit calcium release, further confirming MPT pore opening induced by excess of calcium in the presence of phosphate, as depicted for male rats in the curves of Figure 3.

The role of the MPT in drug-induced organ dysfunction is well documented. However, nimesulide did not promote any early alterations in mitochondrial susceptibility to MPT induction. The result is also in accordance with the observation that the phosphorylation capability of mitochondria isolated from treated animals was not affected. On the basis of in vitro experiments, several investigators have suggested that mitochondrial dysfunction may be involved in the hepatotoxicity induced by nimesulide. Unfortunately, only one of these in vitro studies was performed in the presence of physiological levels of plasma albumin. This is a key factor because nimesulide is $99 \%$ bound to albumin, and only a very small free drug fraction can enter cells and mitochondria. This aspect is intrinsically considered in the present in vivo study. Berson et al. [44] observed that nimesulide uncouples mitochondria and triggers the MPT in isolated mitochondria or hepatoma cells incubated in the absence of albumin. However, in the presence of physiological amounts of albumin, such toxic effects were not observed.

To obviate for the presumably low free drug levels following clinical administration of nimesulide, an experimental protocol using a supratherapeutic dose of the drug was chosen. In spite of these extreme conditions, present data clearly show that nimesulide failed to induce early adverse effects on mitochondria. It is also noteworthy that there is no gender-related predisposition to the potential toxic effects of nimesulide on mitochondrial bioenergetics.

In sequence of the results obtained, we performed additional experiments in three extra male rats exposed for longer times to the drug (animals were treated for 28 days with $50 \mathrm{mg} / \mathrm{kg}$ nimesulide twice daily). The same mitochondrial parameters described in the study were evaluated as rats were killed $2 \mathrm{~h}$ after the last injection. Under these conditions, mitochondria strikingly remained without any noticeable bioenergetic alteration (data not shown). 
The 5-day protocol represents an approach in agreement with the usual therapeutic regimen in humans, that is, 5 days with $100 \mathrm{mg}$ twice daily and discontinuation after. In our protocol, we have followed a washout period of 2 days to confirm whether nimesulide had persistent effects on mitochondria, which was already described with other well-known drugs (e.g., doxorubicin) [34,45].

Another important clue for the lack of effect of in vivo nimesulide treatment on hepatic mitochondrial function derives from the results obtained by Mingatto et al. [18], who clearly demonstrated that one of the nimesulide metabolites (the product of reduction of the nitro group to an amine derivative) does not have the same effects of the parent compound regarding the induction of the permeability transition on isolated mitochondrial fractions.

In conclusion, nimesulide had no early effects on liver mitochondrial function following repeated administration of a largely supratherapeutic dose (50 $\mathrm{mg} / \mathrm{kg}$, twice daily for five consecutive days) to rats. This is in contrast to the positive results obtained in vitro by various authors and may be at least in part due to the absence of physiological levels of albumin in these in vitro experiments and to the hepatic metabolism of nimesulide whereby the drug is converted to less toxic metabolites.

The hypothesis put forward by various investigators that early mitochondrial dysfunction may be linked to very rare clinical cases of nimesulide-induced hepatotoxicity was not confirmed in this study, as hepatic mitochondria from nimesulide-treated rats had their performance unaltered. Our results also emphasize that in vitro studies cannot be easily extrapolated to in vivo systems.

\section{ACKNOWLEDGMENTS}

We thank Helsinn Healthcare SA and especially Sergio Cantoreggi, PhD, ERT, for providing us with nimesulide and for his critical review of the final manuscript.

\section{REFERENCES}

1. Rainsford KD. An analysis from clinico-epidemiological data of the principal adverse events from the COX-2 selective NSAID, nimesulide, with particular reference to hepatic injury. Inflammopharmacology 1998;6:203-221.

2. Traversa G, Bianchi C, Da Cas R, Abraha I, MennitiIppolito F, Venegoni M. Cohort study of hepatotoxicity associated with nimesulide and other non-steroidal antiinflammatory drugs. BMJ 2003;327:18-22.
3. Fossaluzza V, Montagnani G. Efficacy and tolerability of nimesulide in elderly patients with osteoarthritis: double-blind trial versus naproxen. J Int Med Res 1989;17:295-303.

4. Pasquale G, Scaricabarozzi I, D'Agostino R, Taborelli G, Vallarino R. An assessment of the efficacy and tolerability of nimesulide vs paracetamol in children after adenotonsillectomy. Drugs 1993;46(suppl 1):234-237.

5. Weiss P, Mouallem M, Bruck R, Hassin D, Tanay A, Brickman CM, Farfel Z, Bar-Meir S. Nimesulide-induced hepatitis and acute liver failure. Isr Med Assoc J 1999;1:89-91.

6. Sbeit W, Krivoy N, Shiller M, Farah R, Cohen HI, Struminger L, Reshef R. Nimesulide-induced acute hepatitis. Ann Pharmacother 2001;35:1049-1051.

7. Van Steenbergen W, Peeters P, De Bondt J, Staessen D, Buscher H, Laporta T, Roskams T, Desnet V. Nimesulideinduced acute hepatitis: evidence from six cases. J Hepatol 1998;29:135-141.

8. Grignola JC, Arias L, Rondán M, Solá L, Bagnulo. Hepatoxicity associated with nimesulide. Arch Med Int 1998;20:13-18.

9. Merlani G, Oehen HP, Cathomas G, Renner EL, Fox M, Fattinger K, Schneemann M, Kullak-Ublick GA. Fatal hepatoxicity secondary to nimesulide. Eur J Clin Pharmacol 2001;57:321-326.

10. Davis R, Brogden RN. Nimesulide: an update of its pharmacodynamic and pharmacokinetic properties, and therapeutic efficacy. Drugs 1994;48:431-454.

11. Zimmerman HJ. Update of hepatotoxicity due to classes of drugs in common clinical use: non-steroidal drugs, anti-inflammatory drugs, antibiotics, antihypertensives, and cardiac and psychotropic agents. Semin Liver Dis 1990;10:322-338.

12. Robin MA, Le Roy M, Descatoire V, Pessayre D. Plasma membrane cytochromes P450 as neoantigens and autoimmune targets in drug-induced hepatitis. J Hepatol 1997;26(suppl 1):23-30.

13. Rainsford KD, Seabrook RW, Spencer S, Hewson AT. Effects of nimesulide and its metabolites or manufacturing intermediates on the viability and growth of the human hepatoma HepG2 cell line. Life Sci 2001;69:29652973.

14. Wallace KB, Starkov, AA. Mitochondrial targets of drug toxicity. Annu Rev Pharmacol Toxicol 2000;40:353388.

15. Chan K, Truong D, Shangari N, O'Brien PJ. Drug-induced mitochondrial toxicity. Expert Opin Drug Metab Toxicol. 2005;1(4):655-669.

16. Caparroz-Assef SM, Bernani-Amado CA, do Nascimento EA, Kelmer-Bracht AM, Ishii-Iwamoto EL. Effects of the nonsteroidal anti-inflammatory drug nimesulide on energy metabolism in livers from adjuvant-induced arthritic rats. Res Commun Mol Pathol Pharmacol 1998;99:93-116.

17. Moreno-Sanchez R, Bravo C, Vasquez C, Ayala G, Silveira LH, Martinez-Lavin M. Inhibition and uncoupling of oxidative phosphorylation by nonsteroidal antiinflammatory drugs: study in mitochondria, submitochondrial particles, cells, and whole heart. Biochem Pharmacol 1999;57:743-752.

18. Mingatto FE, dos Santos AC, Rodrigues T, Pigoso AA, Uyemura SA, Curti C. Effects of nimesulide and its reduced metabolite on mitochondria. Br J Pharmacol 2000;131:1154-1160. 
19. Caparroz-Assef SM, Salgueiro-Pagadigorria CL, BersaniAmado CA, Bracht A, Kelmer-Bracht AM, Ishii-Iwamoto EL. The uncoupling effect of the nonsteroidal antiinflammatory drug nimesulide in liver mitochondria from adjuvant-induced arthritic rats. Cell Biochem Funct 2001;129:117-124.

20. Mingatto FE, Rodrigues T, Pigoso AA, Uyemura SA, Curti C, Santos AC. The critical role of mitochondrial energetic impairment in the toxicity of nimesulide to hepatocytes. J Pharmacol Exp Ther 2002;303:601-607.

21. Zoratti M, Szabo I. The mitochondrial permeability transition. Biochim Biophys Acta 1995;1241:139-176.

22. Kowaltowski AJ, Vercesi AE. Mitochondrial damage induced by conditions of oxidative stress. Free Radical Biol Med 1999;26:463-471.

23. Vieira HLA, Kroemer G. Pathophysiology of mitochondrial cell death control. Cell Mol Life Sci 1999;56:971976.

24. Tay VKS, Wang AS, Leow KY, Ong MMK, Wong KP, Boelsterli UA. Mitochondrial permeability transition as a source of superoxide anion induced by the nitroaromatic drug nimesulide in vitro. Free Radical Biol Med 2005;39:949-959.

25. Bernareggi A. Clinical pharmacokinetics and metabolism of nimesulide Inflammopharmacology 2001;9:81-89.

26. Custodio JB, Moreno AJ, Wallace KB. Tamoxifen inhibits induction of the mitochondrial permeability transition by $\mathrm{Ca}^{2+}$ and inorganic phosphate. Toxicol Appl Pharmacol 1998;152:10-17.

27. Estabrook R. Mitochondrial respiratory control and polarographic measurement of ADP:O ratios. Methods Enzymol 1967;X:41-57.

28. Kamo N, Muratsugu M, Hongoh R, Kobatake Y. Membrane potential of mitochondria measured with an electrode sensitive to tetraphenyl phosphonium and relationship between proton electrochemical potential and phosphorylation potential in steady state. J Membr Biol 1979;49:105-121.

29. Moreno AJM, Madeira VMC. Mitochondrial bioenergetics as affected by DDT. Biochim Biophys Acta 1991;1060:166-174.

30. Broekemeier K, Dempsey M, Pfeiffer D. Cyclosporin $\mathrm{A}$ is a potent inhibitor of the inner membrane mitochondrial transition in liver mitochondria. J Biol Chem 1989;264:7826-7830.

31. Boelsterli U. Mechanisms of NSAID-induced hepatotoxicity: focus on nimesulide. Drug Safety 2002;25:633-648.

32. Wallace JL. Nonsteroidal anti-inflammatory drugs and gastroenteropathy: the second hundred years. Gastroenterology 1997;112:1000-1006.

33. Elson CO, Sartor BR, Tennyson GS, Riddell RH. Experimental models of inflammatory bowel disease. Gastroenterology 1995;109:1344-1367.

34. Oliveira PJ, Bjork JA, Santos MS, Leino RL, Froberg MK, Moreno AJ, Wallace KB. Carvedilol-mediated an- tioxidant protection against doxorubicin-induced cardiac mitochondrial toxicity. Toxicol Appl Phamacol 2004;200:159-168.

35. Bernareggi A. Clinical pharmacokinetics of nimesulide. Clin Pharmacokinet 1998;35:247-274.

36. Rainsford KD. Nimesulide-a multifactorial approach to inflammation and pain: scientific and clinical consensus. Curr Med Res Opin 2006;22:1161-1170.

37. Hull ML, Prentice A, Wang DY, Wang RP, Butt RP, Phillips SC, Smith SK, Charnock-Jones DS. Nimesulide, a COX-2 inhibitor, does not reduce lesion size or number in nude mouse model of endometriosis. Hum Reprod 2005;20:350-358.

38. Gupta SK, Velpandim T, Mathur P, Sengupta S. Comparative analgesic activity of nimesulide and diclofenac by intramuscular route: correlation with pharmacokinetic profile of nimesulide. Pharmacology 1998;56:137143.

39. Nieminen A-L, Saylor AK, Tesfai SA, Herman B, Lemasters JJ. Contribution of the mitochondrial permeability transition to lethal injury after exposure of hepatocytes to t-butylhydroperoxide. Biochem J 1995;307:99106.

40. Kass GE, Juedes MJ, Orrenius S. Cyclosporin A protects hepatocytes against prooxidant-induced cell killing. A study on the role of mitochondrial $\mathrm{Ca}^{2+}$ cycling in cytotoxicity. Biochem Pharmacol 1992;44:1995-2003.

41. Lemasters JJ, Nieminen AL, Qian T, Trost LC, Elmore SP, Nishimura Y, Crowe RA, Cascio WE, Bradham CA, Brenner DA, Herman B. The mitochondrial permeability transition in cell death: a common mechanism in necrosis, apoptosis and autophagy. Biochim Biophys Acta 1998;1366:177-196.

42. Berson A, Wolf C, Berger V, Fau D, Chachaty C, Fromenty B, Pessayre D. Generation of free radicals during the reductive metabolism of the nitroaromatic compound, nilutamide. J Pharmacol Exp Ther 1991;257:714719.

43. Kowaltowski AJ, Castilho RF, Grijalba MT, Bechara EJH, Vercesi AE. Effect of inorganic phosphate concentration on the nature of inner mitochondrial membrane alterations mediated by $\mathrm{Ca}^{2+}$ ions. A proposed model for phosphate-stimulated lipid peroxidation. J Biol Chem 1996;271:2929-2934.

44. Berson A, Cazanave S, Descatoire V, Tinel M, Grodet A, Wolf C, Feldmann G, Pessayre D. The anti-inflammatory drug, nimesulide (4-nitro-2phenoxymethane-sulfoanilide), uncouples mitochondria and induces mitochondrial permeability transition in human hepatoma cells: protection by albumin. J Pharmacol Exp Ther 2006;318:444-454.

45. Santos DL, Moreno AJM, Leino RL, Froberg MK, Wallace KB. Carvedilol protects against doxorubicin-induced mitochondrial cardiomyopathy. Toxicol Appl Pharmacol 2002;185:218-227. 